\title{
Aquisição Automática de Competências num Ambiente Educacional Ubíquo
}

\author{
Augusto B. Branquinho ${ }^{1}$, Carlos R. Lopes ${ }^{1}$,Fabiano A. Dorça ${ }^{1}$ \\ Márcia A. Fernandes ${ }^{1}$, Renan G. Cattelan ${ }^{1}$ \\ ${ }^{1}$ Faculdade de Computação - Universidade Federal de Uberlândia (UFU) \\ Campus Santa Mônica - Bloco 1B - Sala 1B148 \\ Av. João Naves de Ávila, 2.121 - Bairro Santa Mônica \\ CEP 38400-902 - Uberlândia-MG - Brasil \\ \{augusto, crlopes, fabiano, marcia, renan\}@ufu.br
}

\begin{abstract}
This paper presents a proposal that aims at equipping a ubiquitous computing environment with tools that enables a user to reach a set of competences. The approach makes use of learning objects suited to the user's learning style, which can be automatically determined by the system. In order to achieve a set of competences, a sequence of learning objects should be presented to the user. In this article we show that a sequence of learning objects can be obtained by means of evolutionary strategies and search algorithms. Experiments carried out indicate that better results can be obtained with evolutionary algorithms.
\end{abstract}

Resumo. Este artigo apresenta uma proposta que tem como objetivo dotar um ambiente de computação ubíqua de ferramenta que possibilite prover ao usuário um mecanismo para reforço ou alcance de um conjunto de competências. A abordagem faz uso de objetos de aprendizagem adequados ao seu estilo de aprendizagem, que pode ser determinado automaticamente pelo sistema. Para alcançar um conjunto de competências, faz-se necessário um sequenciamento de objetos de aprendizagem a serem apresentados, o que foi obtido por meio de estratégias evolucionárias e algoritmos de busca. Nos experimentos realizados, mostra-se que melhores resultados podem ser obtidos com algoritmos evolucionários.

\section{Introdução}

Computação ubíqua é conhecida como a terceira onda da computação, a qual visa tornar os dispositivos computacionais mais integrados à vida das pessoas [Weiser 1991]. Sua idéia é auxiliar as pessoas na realização de atividades diárias, de maneira transparente e não intrusiva. No domínio educacional, esse tecnologia pode apoiar instrutores e alunos no processo de ensino-aprendizagem, permitindo acesso ao conhecimento em qualquer lugar e a qualquer momento. Captura e acesso multimídia e ciência de contexto são dois importantes temas em pesquisas de computação ubíqua. Enquanto o primeiro produz automaticamente um grande volume de mídias digitais (por ex., vídeo, áudio, slides, comentários de texto), o segundo oferece meios adequados para integrar e acessar tal conteúdo. Ao fundir os dois conceitos, os usuários podem se beneficiar de ferramentas que lhes permitam recuperar, visualizar e interagir com os artefatos de mídia capturados.

Neste artigo, conceitos de computação ubíqua são explorados no domínio educacional a partir de uma arquitetura de acesso contextual desenvolvida para personalização 
e recomendação de conteúdo multimídia interativo capturado em ambientes educacionais instrumentados. Essa arquitetura utiliza uma abordagem que considera informações de contexto, preferências do usuário e restrições de apresentação, de modo a personalizar a experiência de acesso, adequando-a às necessidades dos usuários. Como estudo de caso, as propostas foram implementadas no Classroom eXperience [Araújo et al. 2013] é uma plataforma de computação ubíqua para captura multimídia concebida para gravar automaticamente as aulas ministradas e disponibilizá-las posteriormente aos alunos.

Um ambiente de computação ubíqua, tal qual o Classroom eXperience, deve ser estendido para incluir maiores funcionalidades de personalização do conteúdo a ser apresentado ao aluno. Dado que se tem um volume enorme de mídias, que em essência são objetos de aprendizagem, torna-se útil ter uma ferramenta que possa fornecer ao aluno objetos de aprendizagem que irão propiciar-lhe atingir determinadas competências. Para isto será necessário um mecanismo que possa gerar uma sequiência de materiais educacionais que, uma vez assimilados, irão fazer com que a competência desejada seja alcançada.

O trabalho descrito neste artigo mostra o processo de planejamento de uma seqüência de conteúdos para atingir um determinado objetivo educacional. Para isto considera-se que os conteúdos serão representados na forma de objetos de aprendizagem. Os objetivos educacionais ou competências a serem atingidas também serão descritos por meio de objetos de aprendizagem. Desta forma ter-se-á um conjuntos de objetos de aprendizagem que deverão ser dispostos numa ordem de apresentação que leve em consideração determinadas restrições. Por exemplo, um objeto de aprendizagem não pode ser apresentado sem que outro, considerado como seu pré-requisito, tenha sido apresentado primeiramente. Para isto, um primeiro esforço para a solução do problema foi feito com computação evolutiva, em particular Algoritmos Genéticos. São levados em consideração o perfil do estudante, caracterizado pelo seu estilo de aprendizagem, e estado cognitivo, como forma de garantir uma personalização do conteúdo.

O artigo está organizado como descrito a seguir. Na Seção 2 são descritos trabalhos relacionados à abordagem apresentada. A Seção 3 apresenta a plataforma Classroom eXperience. A Seção 4 descreve o mecanismo para diagnóstico do estilo de aprendizagem do aluno, necessário para o sequenciamento de objetos de aprendizagem. O processo de sequenciamento do conteúdo usando computação evolutiva é apresentado na Seção 5 e os resultados obtidos aparecem na Seção 6. Na Seção 7, é feita a conclusão do trabalho.

\section{Trabalhos Relacionados}

Adaptatividade desempenha um papel importante na aprendizagem ubíqua, objetivando proporcionar aos estudantes material de aprendizagem adaptado e personalizado, atividades, e informações no lugar e momento certos. Neste sentido, um dos aspectos importantes a ser considerado é a identificação do estilo de aprendizagem do usuário, permitindo que objetos de aprendizagem se adequem ao seu perfil e lhe propiciem, assim, um tratamento personalizado.

Alguns trabalhos recentes têm apresentado propostas para detecção automática de estilos de aprendizagem (EAs) [Graf and Viola 2009, Limongelli et al. 2009]. Essas propostas utilizam sistemas de inferência determinísticos baseados em padrões de comportamento pré-definidos do estudante, de forma que, através de seu comportamento e ações no sistema, possam inferir seus EAs. [Graf et al. 2009, Graf and Viola 2009] discutem 
padrões de comportamento considerados relevantes para identificação de cada EA.

Sistemas de inferência determinísticos, baseados em padrões de comportamento pré-definidos, ignoram importantes considerações relacionadas aos aspectos dinâmicos e não-determinísticos de EA e do comportamento do estudante [Marzano and Kendall 2007, Messick 1976, Felder and Spurlin 2005, Roberts and Erdos 1993]. Nesse contexto, a abordagem ora apresentada apresenta avanços ao considerar, de forma probabilística, os EAs dos estudantes.

Além da determinação do estilo de aprendizagem do aluno, faz-se necessário o uso de mecanismos que gerem atividades que propiciem a satisfação de um dado objetivo educacional ou competência a ser adquirida por um estudante. Na obtenção de uma ou mais sequências podem ser necessários diversos objetos de aprendizagem. Trabalhos desenvolvidos nesse contexto se enquadram dentro do processo de geração automática de currículo. De maneira geral, objetiva-se o sequenciamento de objetos de aprendizagem que propiciem o alcance de uma determinada competência. Trata-se de um problema NPHard [S. and Menai 2011], pois faz-se necessário uma ordenação dos objetos levando-se em conta restrições existentes entre eles.

Várias abordagens e técnicas de sequenciamento de objetos de aprendizagem têm sido propostos na literatura, entre os quais pode-se citar: sequenciamento baseado em regras [De Bra and Calvi 1998]; sequenciamento baseado em planejamento [Limongelli et al. 2009]; sequenciamento baseado em grafos, como encontrado no sistema Intelligent Web Teacher [Sangineto et al. 2008]; sequencimento baseado em redes bayesianas, tal como no sistema BITS [Butz et al. 2004]; sequenciamento baseado em PSO (Particle Swarm Optmization) [de Marcos et al. 2007]; e computação evolutiva [S. and Menai 2011]. No entanto, cada solução tem seus pontos fortes e limitações. A pergunta "Qual é o melhor algoritmo de sequenciamento para usar em um ambiente de aprendizagem?" é difícil de ser respondida, havendo muitas variáveis que podem afetar essa escolha [Limongelli et al. 2010]. Em função disso, optou-se pela abordagem de Computação Evolutiva com o objetivo de identificar seus pontos fortes e limitações e, assim, adequá-la a nossa plataforma de computação ubíqua.

\section{A Plataforma Classroom eXperience}

A plataforma Classroom eXperience (CX) é uma plataforma de captura multimídia concebida para gravação automática de aulas e sua disponibilização posterior aos alunos. Com o CX é possível capturar, armazenar e acessar conteúdo multimídia capturado em salas de aula instrumentadas com lousas eletrônicas, microfones, câmeras de vídeo e projetores, sincronizando os diversos fluxos de mídia capturados por cada dispositivo e gerando documentos hipermídia em diferentes formatos de apresentação. Tal abordagem permite a personalização da apresentação do conteúdo capturado de acordo com as preferências e o contexto de acesso de cada estudante [Araújo et al. 2013].

Sem alterar a dinâmica convencional das aulas, o CX utiliza recursos de computação ubíqua para auxiliar tanto instrutores, no processo de captura de aulas, quanto estudantes, no processo de acesso ao conteúdo ministrado. O CX conta com componentes de software especializados para gravar as atividades educacionais, sincronizar os diferentes fluxos de mídia gerados e disponibilizar o conteúdo através de uma interface amigável. Todos os componentes foram desenvolvidos utilizando a linguagem Java com suporte a 
bibliotecas Web. Além disso, as informações da aplicação, bem como o conteúdo gerado são armazenados em banco de dados PostgreSQL.

\section{Estilos de Aprendizagem}

Um grande número de estudos atesta que a aprendizagem é facilitada se as estratégias pedagógicas estiverem de acordo com os EAs do estudante, tornando o processo de aprendizagem mais efetivo e melhorando consideravelmente sua performance [Graf et al. 2008, Kinshuk et al. 2009, Graf et al. 2009]. Baseado em tal fato, os autores têm desenvolvido uma abordagem que, de forma automática, dinâmica e probabilística, modela um EA do estudante.

A teoria de EAs, que dá suporte ao trabalho sendo desenvolvido, é o modelo de EA proposto por [Felder and Silverman 1988], o Felder-Silverman's Learning Styles Model (FSLSM). O FSLSM se destaca dos demais por combinar os principais modelos de EA, conforme alerta [Kinshuk et al. 2009]. Na abordagem proposta, os EA dos estudantes são armazenados através de distribuições de probabilidades no modelo do estudante (ME), indicando as probabilidades de preferência por cada EA dentro de cada uma das quatro dimensões do FSLSM, chamados aqui de EA probabilísticos $\left(E A_{p}\right)$. Então, tem-se um ME probabilístico em que EA são tratados pelo sistema como probabilidades, e não como certezas. A Tabela 1 apresenta um exemplo de $E A_{p}$ no ME, representando um estudante que provavelmente é Reflexivo, Intuitivo, Verbal e Sequencial.

Tabela 1. Exemplo de Inicialização dos $E A_{p}$

\begin{tabular}{|c|c|c|c|c|c|c|c|}
\hline \multicolumn{7}{|c|}{ EA Probabilísticos $\left(E A_{p}\right)$} \\
\hline \multicolumn{2}{|c|}{ Processamento } & \multicolumn{2}{c|}{ Percepção } & \multicolumn{2}{c|}{ Entrada } & \multicolumn{2}{c|}{ Organização } \\
\hline Ativo & Reflexivo & Sensitivo & Intuitivo & Visual & Verbal & Sequencial & Global \\
\hline 0,28 & 0,72 & 0,09 & 0,91 & 0,45 & 0,55 & 0,82 & 0,18 \\
\hline
\end{tabular}

Os processos de modelagem automática e dinâmica dos $E A_{p}$ do estudante, e de seleção das estratégias de ensino, reforço e remediação, são baseados na seleção dinâmica de uma combinação de estilos de aprendizagem (CEA) que defina uma ação pedagógica apropriada a ser tomada em determinado momento. Uma CEA é uma tupla formada por 4 estilos de aprendizagem, cada um pertencente a uma dimensão do FSLSM, conforme apresenta a Definição 4.1.

Definição 4.1 Combinação de estilos de aprendizagem (CEA)

$C E A=(a, b, c, d \mid a \in D 1, b \in D 2, c \in D 3, d \in D 4)$

considerando:

$D 1=\{\operatorname{Ativo}(A), \operatorname{Reflexivo}(R)\}$

$D 2=\{\operatorname{Sensitivo}(S)$, Intuitivo $(I)\}$

$D 3=\{\operatorname{Visual}(V i), \operatorname{Verbal}(V e)\}$;

$D 4=\{\operatorname{Sequencial}(\operatorname{Seq}), \operatorname{Global}(G)\}$.

Desta forma, existem $16\left(2^{4}\right)$ possíveis CEAs. A cada seção de aprendizagem do curso, seleciona-se estocasticamente uma CEA de acordo com sua probabilidade de preferência pelo estudante, dada por $\mathrm{P}$, de acordo com (1). Consequentemente, a probabilidade da CEA $(A, S, V i, S e q)$ ser selecionada é dada por $P(A, S, V i, S e q)=$ 
$0,28 \times 0,09 \times 0,45 \times 0,82$. A CEA selecionada define a estratégia pedagógica a ser adotada na apresentação do conteúdo do curso durante uma sessão de aprendizagem.

$$
P(a, b, c, d)=P r_{a} \times P r_{b} \times P r_{c} \times P r_{d}
$$

Considera-se, então, que o estudante pode, com maior ou menor probabilidade, pertencer a qualquer uma das 16 categorias pedagógicas dadas pelas 16 CEAs. Então, pode-se dizer que temos uma classificação probabilística e dinâmica dos estudantes, que estocasticamente se enquadram a estas categorias pedagógicas ao longo do processo de aprendizagem com maior ou menor probabilidade. Com isto, espera-se produzir um melhor tratamento da incerteza inerente à inferência da categoria pedagógica à qual um estudante pertence.

O processo de modelagem automática e dinâmica de um EA é implementado por um algoritmo de aprendizagem por reforço cuja especificação é dada a seguir.

- Estados $(S)$ : Valores de $E A_{p}$ e nível cognitivo armazenados no ME;

- Ações $(A)$ : Ações que o sistema pode executar, com intuito de ensinar o conteúdo maximizando a qualidade da performance do estudante, durante o processo de aprendizagem, ou seja, as CEAs dadas pela Definição 4.1;

- Percepção do ambiente $(I: S \rightarrow S)$ : Indica como o sistema percebe o estado do estudante. Por exemplo, um SAIE pode perceber o estado cognitivo do estudante através da avaliação de seu conhecimento por meio de testes ou exames;

- Reforço ( $R: S \times A \rightarrow \Re)$ : Essa função define os sinais de reforço a serem aplicados no aprendizado das melhores CEAs para um estudante;

- Função valor-ação, ou ganho $(Q: S \times A \rightarrow \Re)$ : Esta função estima a utilidade de se adotar determinada CEA (ação), considerando determinado estado $\left(E A_{p}\right)$, fornecendo um método de avaliação para as ações do sistema. Esta função é dada por (1), e associa uma ação a um número real, denominado ganho, e estima quão boa determinada CEA é em determinado estado.

Maiores detalhes sobre o algoritmo podem ser obtidos em [Dorça et al. 2012].

\section{Objetos de Aprendizagem e Sequenciamento: Conceitos e Aspectos Metodológicos}

Neste trabalho considera-se que objetos de aprendizagem podem representar competências a serem alcançadas e são adequados ao perfil do usuário com base no seu estilo de aprendizagem obtido de acordo com o procedimento especificado na 4. Dado que geralmente teremos um conjunto de competências a serem adquiridas, isto significa que será necessário que o usuário trabalhe com um conjunto de objetos de aprendizagem. Temse então o chamado problema do sequenciamento dos objetos de aprendizagem. Dado uma sequência aleatória de objetos de aprendizagem, o problema consiste em determinar uma sequência ordenada dos mesmos levando-se em conta as restrições existentes. $\mathrm{O}$ uso de objetos de aprendizagem para representar competências e as possíveis restrições existentes entre as mesmas, bem como a técnica usada para resolver o problema do sequenciamento são apresentados nas subseções seguintes. 
CBIE-LACLO 2015

Anais do XXVI Simpósio Brasileiro de Informática na Educação (SBIE 2015)

\subsection{Competências}

O trabalho desenvolvido foi estabelecido levando-se em conta as competências a serem adquiridas pelo usuário. As competências podem ser descritas como "entidades multidimensionais constituídas por conhecimento, habilidades e fatores psicológicos que surgem de forma integrada como um comportamento em função de uma interação com o ambiente" [García et al. 2010].

Existem diversas iniciativas objetivando padronizar a definição de competência de tal modo que ela poderia ser feita por máquinas. Por exemplo, o comitê IEEE-LSTC define que padrão para uma tecnologia de aprendizagem corresponde a um padrão para definicão de competências que podem ser reusadas. Ainda de acordo com a nomenclatura IEEE, um registro de competência é chamado de Definição de Competência Reusável. A definição de uma competência reusável pode ser feita, num nível mínimo, pela sequência de dois objetos de aprendizagens: um estabelecendo objetivos educacionais a serem alcançados e outro correspondendo um pré-requisito a ser satisfeito. Informações especificando objetivos, pré-requisitos e outros dados de interesse são denominados de metadados dos objetos de aprendizagem e devem aparecer na especificação dos objetos. No trabalho que foi desenvolvido, adotou-se o padrão LOM (Learning Object Metadata) para especificação de metadados. Esse padrão é caracterizado pela especificação de uma série de registros que descrevem os metadados. Maiores detalhes sobre metadados relativos ao LOM podem ser obtidos em [IEEE 2010].

Em função do exposto, tem-se que o objetivo a ser alcançado é fazer com que o usuário assimile um conjunto de competências. Assim sendo, teremos um conjunto de objetos de aprendizagem não ordenados que correspondem às competências desejadas. Um cuidado deve ser tomado ao se apresentar os objetos de aprendizagem aos usuários: não é conveniente apresentar um determinado objeto de aprendizagem sem antes apresentar outro(s) que correspondam aos seus pré-requisitos. Desta forma, torna-se necessária uma correta ordenação ou sequenciamento dos objetos, que é a solução para o problema do sequenciamento de objetos. Neste trabalho, foram utilizadas duas técnicas para resolver o problema do sequenciamento: estratégias evolucionárias e algoritmos de busca.

\subsection{Estratégias Evolucionárias}

Para realizar o planejamento sequencial dos objetos de aprendizado foram utilizadas estratégias evolucionárias. Essas estratégias têm como base o processo de evoluir as soluções com base em gerações. O processo consiste em um conjunto de passos. Inicialmente é realizada a geração aleatória da população inicial. A população consiste em um grupo de indivíduos, cujo tamanho pode variar de acordo com os experimentos. Cada indivíduo corresponde a uma possível solução de um dado problema. Após gerar a população inicial, são executados métodos de evolução. A evolução, também chamada de geração, consiste primeiramente na seleção de indivíduos da população. São selecionados pares de indivíduos (pais), e, utilizando o processo de crossover e mutação, são gerados novos indivíduos (filhos). Após a geração das novas soluções, é realizada a seleção natural. Este procedimento é responsável por eliminar as piores soluções, mantendo a população sempre com o mesmo tamanho e com os melhores indivíduos.

Para o problema dos objetos de aprendizado, cada indivíduo corresponde a uma sequência de objetos de aprendizado ordenados de acordo com as restrições e competên- 
cias. Muitos indivíduos possuem restrições que podem não ser satisfeitas pela sequência de objetos. Desse modo, o objetivo é que as estratégias encontrem as sequências que satisfarão todas as restrições.

Para avaliar o quão bom é um indivíduo, é realizado o cálculo da aptidão. Essa aptidão, comumente chamada de fitness, tem como base as restrições e a posição de cada objeto de aprendizado. O fitness do trabalho é expresso pela equação fitness $(s)=$ $\sum_{i=1}^{n} i * \operatorname{pr}\left(s_{i}\right)$ [García et al. 2010], onde a variável $s$ corresponde a uma sequência de objetos e $\operatorname{pr}\left(s_{i}\right)$ é o total de requisitos não satisfeitos para o objeto na posição $i$.

A Tabela 2 mostra uma sequência com 6 objetos e seus respectivos requisitos e competências. Cada competência foi representada por uma letra. Neste caso o objeto $\mathrm{LO}_{1}$ não possui nenhum requisito. Já $\mathrm{LO}_{2}$ tem seu requisito satisfeito pelo resultado de $\mathrm{LO}_{1}$. Em seguida, $\mathrm{LO}_{3}$ possui os requisitos $\mathrm{E}$ e $\mathrm{F}$ que não são satisfeitos. Esse processo é realizado para cada objeto e ao final a aptidão é dada por fitness $(s)=1 * 0+2 * 0+3 *$ $2+4 * 0+5 * 1+6 * 0=11$.

Tabela 2. Sequência de objetos com as respectivas restrições e competências.

\begin{tabular}{|c|c|c|c|c|c|c|}
\hline Sequência de Objetos & $\mathrm{LO}_{1}$ & $\mathrm{LO}_{2}$ & $\mathrm{LO}_{3}$ & $\mathrm{LO}_{4}$ & $\mathrm{LO}_{5}$ & $\mathrm{LO}_{6}$ \\
\hline Requisitos & & B & C, E, F & C, D & F, K & G \\
\hline Competências & B & C, D & G & F & H & K \\
\hline
\end{tabular}

Este trabalho utiliza duas abordagens evolucionárias, sendo o algoritmo genético tradicional e o algoritmo steady state [Luke 2013]. A diferença fundamental desses procedimentos é que, enquanto o algoritmo genético seleciona vários pares de indivíduos durante uma evolução, o steady state escolhe apenas um par. O objetivo do steady state é que ele exerça uma pressão seletiva maior [Luke 2013]. Outro ponto que diverge entre esses métodos é que o algoritmo genético possui como parâmetro de parada um número fixo de gerações e o steady state pára de executar após um número de iterações sem evolução.

Para realizar a seleção dos indivíduos que sofreram crossover, foram usadas três técnicas, sendo por roleta, torneio e aleatório. A roleta realiza uma seleção de acordo com um sorteio considerando o percentual de quão bom são os indivíduos. Já o torneio necessita de um parâmetro inicial $n$ que determina quanto elementos serão inicialmente selecionados através da roleta. Uma vez escolhidos os $n$ indivíduos o melhor dentre eles é dado como resultado do torneio. Por último, o método aleatório apenas escolhe de forma aleatória os indivíduos.

Existem diversos métodos de crossover, sendo que neste trabalho foi usado o PMX. O crossover PMX consiste em selecionar uma região de cada indivíduo e, posteriormente, é realizada a troca dos objetos dentro do intervalo da região selecionada. A Figura 1 exibe o crossover PMX.

A partir de dois indivíduos, chamados de pais, são gerados dois novos filhos. No primeiro passo é selecionada um intervalo de objetos de cada pai. No segundo passo é realizada a troca dos objetos dentro do intervalo. Posteriormente, no passo 3, são verificados quais objetos se repetem para cada indivíduo. Ao final, é realizada mais uma troca dos objetos repetidos seguindo a mesma sequência em que ocorreram as repetições.

Cada indivíduo possui uma probabilidade de ocorrer mutação. Essa probabilidade 


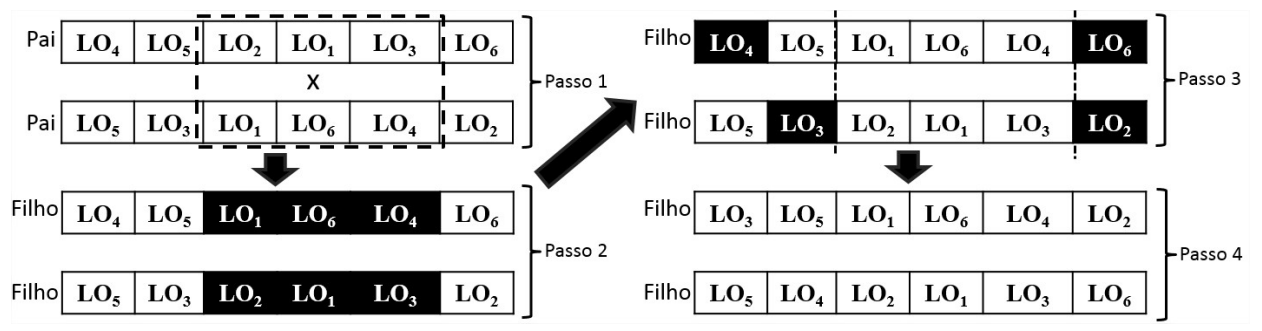

Figura 1. Passo a passo do crossover PMX.

é definida no início da execução. Neste trabalho, a mutação realiza uma simples troca de posição de um objeto. Após a mutação todos os novos indivíduos são adicionados a população atual. Posteriormente, a seleção natural remove os piores indivíduos com o objetivo de manter a população com o mesmo tamanho inicial. Ao final, se a solução ótima foi encontrada ou o critério de parada foi satisfeito, o melhor indivíduo é dado como solução. É importante lembrar que o critério de parada para o algoritmo genético e o steady state são, respectivamente, o número de gerações e o limite de iterações sem melhoria da solução.

\section{Resultados}

Para avaliar a aplicação das estratégias evolucionárias e dos algoritmos de busca, foram realizados vários experimentos. Cada experimento foi executado 100 vezes e o resultado final é dado pela média dos resultados. Foram utilizados 9 diferentes conjuntos de objetos, sendo que cada grupo possui, respectivamente, as quantidades de 10, 20, 30, 40, 50, $60,70,80$ e 90 objetos. Esses objetos foram gerados aleatoriamente com o número de restrições e competência variando de 0 a 4.

Os experimentos foram separados em quatro grupos. Em cada grupo foram alterados os parâmetros com o objetivo de buscar melhores resultados. O primeiro grupo utilizou o algoritmo genético tradicional com população contendo 50 indivíduos. $\mathrm{O}$ critério de parada consiste em encontrar o fitness ótimo (igual a 0) ou alcançar o número de gerações. O máximo de gerações foram de 100 e 200. Para o processo de seleção foram usados os métodos de roleta, aleatório e torneio 3. Foi usada uma mutação com probabilidades de $4 \%, 10 \%$ e $20 \%$. No segundo grupo foram utilizados os mesmo parâmetros do primeiro grupo, sendo alterado apenas para o algoritmo steady state. Já no terceiro grupo foi aplicado o algoritmo de busca Hillclimb. Por último, o quarto grupo utilizou o algoritmo de buscar $\mathrm{A}^{*}$.

Para avaliar os experimentos foram considerados os valores de fitness e o tempo necessário para geração das soluções. Dentre os algoritmos de busca, o Hillclimb foi aquele que encontrou os melhores resultados. Já considerando as técnicas evolutivas, os melhores resultados foram obtidos ao utilizar o algoritmo genético tradicional com 50 indivíduos, 200 gerações, seleção por roleta 3, crossover PMX e taxa de mutação de $20 \%$. Comparando as técnicas de busca e algoritmo evolutivo, de modo geral os melhores resultados foram obtidos pelo algoritmo evolutivo. A Figura 2 mostra, respectivamente, os resultado do Fitness e tempo para geração das soluções comparando a busca Hillclimb e o algoritmo evolutivo. 


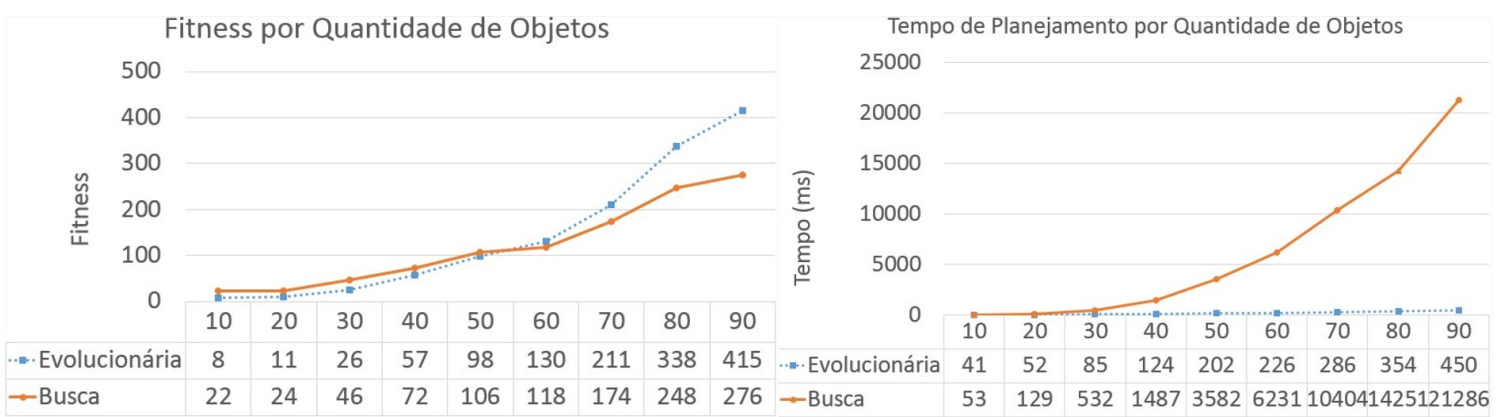

Figura 2. Fitness e tempo de processamento de acordo com a quantidade de objetos de aprendizagem.

\section{Conclusão}

A abordagem descrita neste artigo representa um esforço inicial de se integrar a um ambiente educacional ubíquo um processo de sequenciamento automático de objetos de aprendizagem de modo a permitir ao aluno a aquisição de determinadas competências. Os objetos de aprendizagem que serão apresentados ao aluno levam em consideração seu estilo de aprendizagem, que pode ser obtido dinamicamente. Na solução do problema de sequenciamento de objetos considerou-se estratégias evolucionárias e uma técnica de busca e, pelos experimentos realizados, chegou-se a resultados melhores fazendo uso de um algoritmo evolucionário steady-state. Trabalhos futuros serão realizados no sentido de se consolidar os algoritmos delineados à plataforma $\mathrm{CX}$, obter melhores funções de fitness bem como explorar novas técnicas de sequenciamento de objetos de aprendizagem.

\section{Agradecimentos}

Os autores agradecem apoio proveniente da Fundação de Amparo a Pesquisa do Estado de Minas Gerais (FAPEMIG), do Programa PET/SESu/MEC e do Programa de Pósgraduação em Ciência da Computação da Universidade Federal de Uberlândia.

\section{Referências}

Araújo, R., Brant-Ribeiro, T., Cattelan, R., De Amo, S., and Ferreira, H. (2013). Personalization of interactive digital media in ubiquitous educational environments. In IEEE International Conference on Systems, Man, and Cybernetics (SMC), pages 3955-3960.

Butz, C., Hua, S., and Maguire, R. (2004). A web-based intelligent tutoring system for computer programming. In IEEE/WIC/ACM Conference on Web Intelligence (WIO4).

De Bra, P. and Calvi, L. (1998). AHA! An open adaptive hypermedia architecture. New Review of Hypermedia and Multimedia, 4(1):115-139.

de Marcos, L., Pages, C., Martinez, J., and Gutierrez, J. (2007). Competency-based learning object sequencing using particle swarms. In 19th IEEE International Conference on Tools with Artificial Intelligence (ICTAI).

Dorça, F., Lima, L., Fernandes, M., and Lopes, C. (2012). Avaliação de políticas de aprendizagem por reforço para modelagem automática e dinâmica de estilos de aprendizagem: Uma análise experimental. In Simpósio Brasileiro de Informática na Educação (SBIE). 
CBIE-LACLO 2015

Anais do XXVI Simpósio Brasileiro de Informática na Educação (SBIE 2015)

Felder, R. and Silverman, L. (1988). Learning and teaching styles in engineering education. Journal of Engineering education, 78(7):674-681.

Felder, R. and Spurlin, J. (2005). Applications, reliability and validity of the index of learning styles. International Journal of Engineering Education, 21(1):103-112.

García, A., de Marcos, L., García, E., and Gutiérrez, J. A. (2010). Courseware sequencing using heuristic and local search. In Arabnia, H. R., Bahrami, A., and Solo, A. M. G., editors, CSREA EEE, pages 70-75. CSREA Press.

Graf, S., Kinshuk, and Liu, T. (2009). Supporting teachers in identifying students' learning styles in learning management systems: An automatic student modelling approach. Journal of Educational Technology \& Society, 12(4):3-14.

Graf, S., Liu, T.-C., and Kinshuk (2008). Interactions Between Students Learning Styles, Achievement and Behaviour in Mismatched Courses. In Proceedings of the International Conference on Cognition and Exploratory Learning in Digital Age (CELDA 2008), pages 223-230. IADIS International Conference.

Graf, S. and Viola, S. (2009). Automatic student modelling for detecting learning style preferences in learning management systems. Citeseer.

IEEE (2010). LOM (Learning Object Metadata). IEEE Learning Technology Standards Committee, http://ltsc.ieee.org/wg12/index.html.

Kinshuk, Liu, T., and Graf, S. (2009). Coping with Mismatched Courses: Students' behaviour and performance in courses mismatched to their learning styles. Educational Technology Research and Development, 57(6):739-752.

Limongelli, C., Sciarrone, F., Temperini, M., and Vaste, G. (2009). Adaptive learning with the LS-plan system: a field evaluation. IEEE Transactions on Learning Technologies, pages 203-215.

Limongelli, C., Sciarrone, F., Temperini, M., and Vaste, G. (2010). Comparing curriculum sequencing algorithms for intelligent adaptive (e)-learning. In Proceedings of the Conference ICL.

Luke, S. (2013). Essentials of Metaheuristics. Lulu, 2 edition.

Marzano, R. and Kendall, J. (2007). The new taxonomy of educational objectives. Corwin Pr.

Messick, S. (1976). Personal styles and educational options. Individuality in learning, pages 327-368.

Roberts, M. and Erdos, G. (1993). Strategy selection and metacognition. Educational Psychology, 13(3):259-266.

S., A.-M. and Menai, M. (2011). Evolutionary computation approaches to the curriculum sequencing problem. Natural Computing, 10(2):891-920.

Sangineto, E., Capuano, N., Gaeta, M., and Micarelli, A. (2008). Adaptive course generation through learning styles representation. Universal Access in the Information Society, 7(1):1-23.

Weiser, M. (1991). The Computer for the 21st Century. Scient. American, 265(3):66-75. 